\title{
Research and Application of Multi-Target Tracking Based on GM-PHD Filter
}

\author{
Yanyi Li, Limin Guo, Xiangsong Huang \\ Harbin Engineering University, Harbin, China \\ Email:925107836@qq.com, guolimin@hrbeu.edu.cn, huangxiangsong@hrbeu.edu.cn
}

How to cite this paper: Li, Y.Y., Guo, L.M. and Huang, X.S. (2020) Research and Application of Multi-Target Tracking Based on GM-PHD Filter. Optics and Photonics Journal, 10, 125-133. https://doi.org/10.4236/opj.2020.106013

Received: April 27, 2020

Accepted: June 26, 2020

Published: June 29, 2020

\begin{abstract}
In recent years, multi-target tracking technology based on Gaussian MixtureProbability Hypothesis Density (GM-PHD) filtering has become a hot field of information fusion research. This article outlines the generation and development of multi-target tracking methods based on GM-PHD filtering, and the principle and implementation method of GM-PHD filtering are explained, and the application status based on GM-PHD filtering is summarized, and the key issues of the development of GM-PHD filtering technology are analyzed.
\end{abstract}

\section{Keywords}

GM-PHD, Multi-Target Tracking, Random Finite Set

\section{Introduction}

In traditional multi-target tracking, the distribution between observation data and targets is realized by data correlation technology, and then the target state estimation is realized by filtering technology. Typical data association target tracking methods, such as nearest neighbor, joint probabilistic data association, these algorithms, have two main problems [1]. First, when the data of the core process is related to the operation, when the number of targets gradually increases, there is a combination of explosions, and the amount of calculation increases exponentially. The second is that when multiple targets are tracked, it is usually assumed that the number of all targets keeps the same, but this condition is often not met in reality.

Previously, the multi-target tracking method based on random finite set theory found by Mahler has become a popular technology for the development of multi-target tracking technology, because it could effectively avoid the prob- 
lems caused by the correlation of traditional data and at the same time it can realize the estimation of variable target number. In 2003, Mahler first proposed the standard Probability Hypothesis Density (PHD) filter. Because of the complex high-dimensional integration operation in the methods of solving PHD, the promotion and application of PHD is limited, and many scholars try to find a suitable method to implement the PHD filter. Ba-Ngu Vo and Wing-Kin Ma proposed a GM-PHD filter based on the linear Gaussian hypothesis, which approximates PHD with the sum of multiple Gaussian terms [2]. It is assumed that the detection probability and survival probability are independent with the states, and the PHD of the target derivative set and the new set can be both expressed as Gaussian sum form, the analytical solution of PHD is obtained by updating the prediction of PHD, and the state information of each Gaussian item can be obtained, and the calculation amount due to the increase of Gaussian item is reduced by pruning and merging strategy. Target tracking has less computational complexity, and has attracted the attention of many scholars and engineering technicians.

This paper reviews the development process of multi-target tracking method based on the Gaussian Mixture-Probability Hypothesis Density (GM-PHD) filtering, discusses the current development status of the technology, and discusses the future improvement and development trend of GM-PHD filtering technology.

\section{GM-PHD Filter}

\subsection{Basic Principles of PHD Filters}

The PHD filter algorithm is actually a first-order moment recursion process for the posterior multi-target state. Because the integration step of the probability density function is completed on the motion state of a single target, it has a small amount of calculation and compared with the Bayes filter algorithm, it is easier to handle. The process of PHD filtering is the same as the traditional Bayes filtering. It is also divided into a forecasting process and an update. The core thought process is as Figure 1.

$X_{k}=\left\{X_{k, 1}, \cdots, X_{k, N_{k}}\right\}$ represents the set of random set states at time $k$, Where $N_{k}$ is the number of targets at time $k, Z_{k}=\left\{Z_{k, 1}, \cdots, Z_{k, M_{k}}\right\}$ represents the random set observation set at time $k$, Where $M_{k}$ is the number of observations at time $k, Z^{(k)}: Z_{1}, \cdots, Z_{k}$ represents the random set observation set at time $k$.

$D_{k \mid k}\left(x \mid Z^{(k)}\right)$ represents the density function at time $k$ corresponding to the multi-target posterior density $p\left(X_{K} \mid Z^{(k)}\right)$, equivalent to the first moment approximation of the multi-objective posterior density.

The prediction equation of PHD filtering can be expressed as:

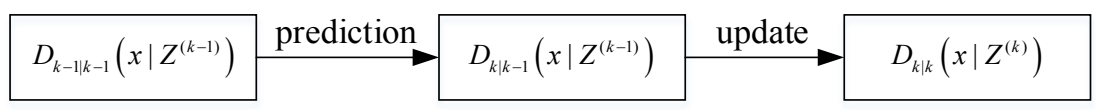

Figure 1. PHD algorithm schematic. 


$$
D_{k \mid k-1}\left(x \mid Z^{(k-1)}\right)=\int \phi_{k \mid k-1}\left(X, X_{k-1}\right) D_{k-1 \mid k-1}\left(x \mid Z^{(k-1)}\right) d X_{k-1}+\gamma_{k}(X)
$$

where $\phi_{k \mid k-1}(x, \xi)=b_{k \mid k-1}(x \mid \xi)+e_{k \mid k-1}(\xi) f_{k \mid k-1}(x \mid \xi), \quad b_{k \mid k-1}(\cdot \mid \xi)$ Represents the PHD of the derivative target set $B_{k \mid k-1}(\{\xi\}), e_{k \mid k-1}(\cdot)$ represents the probability of existence of target, $f_{k \mid k-1}(\cdot \mid \cdot)$ represents the transition probability density of a single target, $\gamma_{k}(\cdot)$ is the PHD of the newly generated target set $\Gamma_{k}$.

The update equation of PHD filtering can be shown as:

$$
\left\{\begin{array}{l}
D_{k \mid k}\left(x \mid Z^{(k)}\right)=L_{z}(x) D_{k-1 \mid k-1}\left(x \mid Z^{(k-1)}\right) \\
D_{k \mid k-1}\left[P_{D, k} L_{Z}\right]=\int P_{D, k}(x) L_{Z_{k}}(x) D_{k \mid k-1}\left(x \mid Z^{(k-1)}\right) d x
\end{array}\right.
$$

where $L_{z_{k}}(x)$ is the observed likelihood function of the single target, the expression is as Equation (3), $\lambda_{k}$ is the clutter intensity, $c_{k}(z)$ is the spatial distribution of the clutter, and $\lambda_{k} c_{k}(z)$ is the PHD of the random set of clutter. $P_{D, k}(\cdot)$ represents detection probability.

$$
L_{z_{k}}(x)=1-P_{D, K}(x)+\sum_{z \in Z_{k}} \frac{P_{D, K}(x) L_{z_{z}}(x)}{\lambda_{k} c_{k}(z)+D_{k \mid k-1}\left[P_{D, K} L_{z}\right]}
$$

In a multi-target tracking environment, the updated PHD function $D_{k \mid k}\left(x \mid Z^{(k)}\right)$ integral could be expressed as

$$
N_{k}=\int D_{k \mid k}\left(x \mid Z^{(k)}\right) d x
$$

Taking the integer value closest to $N_{k}$ as the desired result of the target number at time $k$, estimated value of the target number could be expressed as Equation (2), and the peak number indicates the target number.

$$
\hat{N}_{k}=\left[N_{k}\right]_{\text {int }}
$$

\subsection{Principle of GM-PHD Filter}

There are complex integration operations in the PHD filter update process, which limits the promotion and application of PHD filter. At present, there are two main implementation methods: one is the sequential Monte Carlo method; the other is the Gaussian mixture method. Based on the assumption of linear Gaussian, Vo proposed a GM-PHD filtering algorithm [3]. The algorithm approximates the multi-object PHD in the form of Gaussian sum. Assuming that the detection probability and survival probability are independent of the state, and that the PHD of the derived target finite set and the new target finite set have Gauss sum, then, suppose the following three conditions are met, the analytical solution of the PHD recursion formula can be obtained by predicting and updating the PHD [4].

The first condition is both the target motion model $f_{k \mid k-1}(\mathbf{x} \mid \zeta)$ and the sensor measurement model $g_{k}(\mathbf{z} \mid \mathbf{x})$ are linear Gaussian models:

$$
\left\{\begin{array}{l}
f_{k \mid k-1}(\mathbf{x} \mid \zeta)=\mathcal{N}\left(\mathbf{x} ; \mathbf{F}_{k-1} \zeta, \mathbf{Q}_{k-1}\right) \\
g_{k}(\mathbf{z} \mid \mathbf{x})=\mathcal{N}\left(\mathbf{z} ; \mathbf{H}_{k} \mathbf{x}, \mathbf{R}_{k}\right)
\end{array}\right.
$$

where $\mathcal{N}(\cdot ; \mathbf{m}, \mathbf{P})$ represents a Gaussian distribution with mean density $m$ and 
variance $P, F_{k-1}$ is the matrix of state transition, $Q_{k-1}$ is the matrix of system noise covariance, $H_{k}$ is the observation matrix, $R_{k}$ is the measurement noise covariance matrix.

The second condition is to assume that the target survival probability and sensor detection probability are independent with the target state:

$$
\left\{\begin{array}{l}
p_{S, k}(x)=p_{S, k} \\
p_{D, k}(x)=p_{D, k}
\end{array}\right.
$$

The third condition is that the strength of the new target random set $\gamma_{k}(\mathbf{x})$ and a derivative target random set $\beta_{k \mid k-1}(\mathbf{x} \mid \zeta)$ are both Gaussian mixed forms:

$$
\left\{\begin{array}{l}
\gamma_{k}(\mathbf{x})=\sum_{i=1}^{J_{\gamma, k}} \omega_{\gamma, k}^{(i)} \mathcal{N}\left(\mathbf{x} ; \mathbf{m}_{\gamma, k}^{(i)}, \mathbf{P}_{\gamma, k}^{(i)}\right) \\
\beta_{k \mid k-1}(\mathbf{x} \mid \zeta)=\sum_{j=1}^{J_{\beta, k}} \omega_{\beta, k}^{(j)} \mathcal{N}\left(\mathbf{x} ; \mathbf{F}_{\beta, k-1}^{(j)} \zeta+\mathbf{d}_{\beta, k-1}^{(j)}, \mathbf{Q}_{\beta, k-1}^{(j)}\right)
\end{array}\right.
$$

where $J_{\gamma, k}^{(i)}, \quad \omega_{\gamma, k}^{(i)}, m_{\gamma, k}^{(i)}, \quad p_{\gamma, k}^{(i)}, i=1,2, \ldots, J_{\gamma, k}$ and other parameters determine the strength of the newly generated target random set, $\omega_{\gamma, k}^{(i)}, m_{\gamma, k}^{(i)}, p_{\gamma, k}^{(i)}$ are the weight, mean and covariance of the $i$ th Gaussian component of the newly generated target random set strength, $J_{\gamma, k}$ is the number of new target Gaussian components at time $k$, similarly, $J_{\beta, k}, \omega_{\beta, k}^{(j)}, \mathbf{d}_{\beta, k-1}^{(j)}, \mathbf{Q}_{\beta, k-1}^{(j)}, j=1,2, \ldots, J_{\beta, k}$ other parameters determines the strength of the target random set derived from the target, $\omega_{\beta, k}^{(j)}, \mathbf{d}_{\beta, k}^{(j)}, \mathbf{Q}_{\beta, k}^{(j)}$, are the weight, mean and covariance of the ith Gaussian component of the newly generated target random set strength, $J_{\beta, k}$ is the number of new target Gaussian components.

Assuming that the posterior PHD at time $k-1$ is Gaussian, the predicted PHD at time $k$ and the posterior PHD at $k$ are also Gaussian mixtures. Then Ma and Vo obtained the analytical expression form of PHD filter, GM-PHD filter through Gaussian mixing technology. It mainly includes the following four steps:

The first step is the prediction process, assume that the posterior intensity at time $k-1$ has the following mixed Gaussian form:

$$
D_{k-1 \mid k-1}\left(\mathbf{x}_{k-1} \mid Z_{1: k-1}\right)=\sum_{i=1}^{J_{k-1}} \omega_{k-1}^{(i)} \mathcal{N}\left(\mathbf{x}_{k-1} ; \mathbf{m}_{k-1}^{(i)}, \mathbf{P}_{k-1}^{(i)}\right)
$$

Then the predicted PHD at time $k$ can be shown as a Gaussian mixture:

$$
D_{k \mid k-1}\left(\mathbf{x}_{k} \mid Z_{1: k-1}\right)=D_{S, k \mid k-1}\left(\mathbf{x}_{k} \mid Z_{1: k-1}\right)+D_{\beta, k \mid k-1}\left(\mathbf{x}_{k} \mid Z_{1: k-1}\right)+\gamma_{k}\left(\mathbf{x}_{k}\right)
$$

The second step is the update process, suppose the predicted PHD at time $k$ could be described as a Gaussian mixture:

$$
D_{k \mid k-1}\left(\mathbf{x}_{k} \mid Z_{1: k-1}\right)=\sum_{i=1}^{J_{k k-1}} \omega_{k \mid k-1}^{(i)} \mathcal{N}\left(\mathbf{x}_{k} ; \mathbf{m}_{k \mid k-1}^{(i)}, \mathbf{P}_{k \mid k-1}^{(i)}\right)
$$

Then the posterior PHD at time $k$ is also Gaussian mixture:

$$
D_{k \mid k}\left(\mathbf{x}_{k} \mid Z_{1: k}\right)=\left(1-p_{D, k}\right) D_{k \mid k-1}\left(\mathbf{x}_{k} \mid Z_{1: k}\right)+\sum_{z_{k} \in Z_{k}} D_{D, k}\left(\mathbf{x}_{k} \mid \mathbf{z}_{k}\right)
$$


where $\left\{\begin{array}{l}D_{D, k}\left(\mathbf{x}_{k} \mid \mathbf{z}_{k}\right)=\sum_{j=1}^{J_{k k-1}} \omega_{k}^{(j)}(\mathbf{z}) \mathcal{N}\left(\mathbf{x}_{k} ; \mathbf{m}_{k \mid k}^{(j)}(\mathbf{z}), \mathbf{P}_{k \mid k}^{(j)}\right) \\ \omega_{k}^{(j)}(z)=\frac{p_{D, k} \omega_{k \mid k-1}^{(j)} q_{k}^{(j)}(z)}{k_{k}(z)+p_{D, k} \sum_{l=1}^{J_{k k-1}} \omega_{k \mid k-1}^{(l)} q_{k}^{(l)}(z)} \\ \mathbf{m}_{k \mid k}^{(j)}(\mathbf{z})=\mathbf{m}_{k \mid k-1}^{(j)}+\mathbf{K}_{k}^{(j)}\left(\mathbf{z}-\mathbf{H}_{k} \mathbf{m}_{k \mid k-1}^{(j)}\right) \\ \mathbf{P}_{k \mid k}^{(j)}=\left[\mathbf{I}-\mathbf{K}_{k}^{(j)} \mathbf{H}_{k}\right] \mathbf{P}_{k \mid k-1}^{(j)} \\ \mathbf{K}_{k}^{(j)}=\mathbf{P}_{k \mid k-1}^{(j)} \mathbf{H}_{k}^{T}\left(\mathbf{H}_{k} \mathbf{P}_{k \mid k-1}^{(j)} \mathbf{H}_{k}^{T}+\mathbf{R}_{k}\right)^{-1}\end{array}\right.$.

The third step is pruning and merging: The Gaussian mixing term of the GM-PHD filter will continue to grow. To solve this problem, the literature adopts the idea of pruning and merging, deletes the Gaussian terms with smaller weights, and merges the Gaussian terms that are closer together.

The fourth step is state extraction: Obtaining the number of targets: by mixing the weights of the Gaussian items and obtaining the number of targets in a monitoring area:

$$
\hat{n}_{k}=\sum_{i=1}^{J_{k}} \tilde{\omega}_{k}^{(i)}
$$

Obtain the target state: extract the Gaussian component with a weight greater than $\tau\left(\tau \geq \frac{1}{2}\right)$ :

$$
\hat{X}_{k}=\left\{\widetilde{\mathbf{m}}_{k}^{(i)}: \tilde{\omega}_{k}^{(i)}>\tau\right\}
$$

\subsection{GM-PHD Filter Simulation}

GM-PHD could be widely utilized in the field of multi-targets. Figure 2 shows that the definition of GM-PHD in the case of two targets as follows:

The simulation conditions are defined as follows: Initially, the A target coordinates are $(250,250)$, the $B$ target coordinates are $(-250,-250)$, and the $A$ and

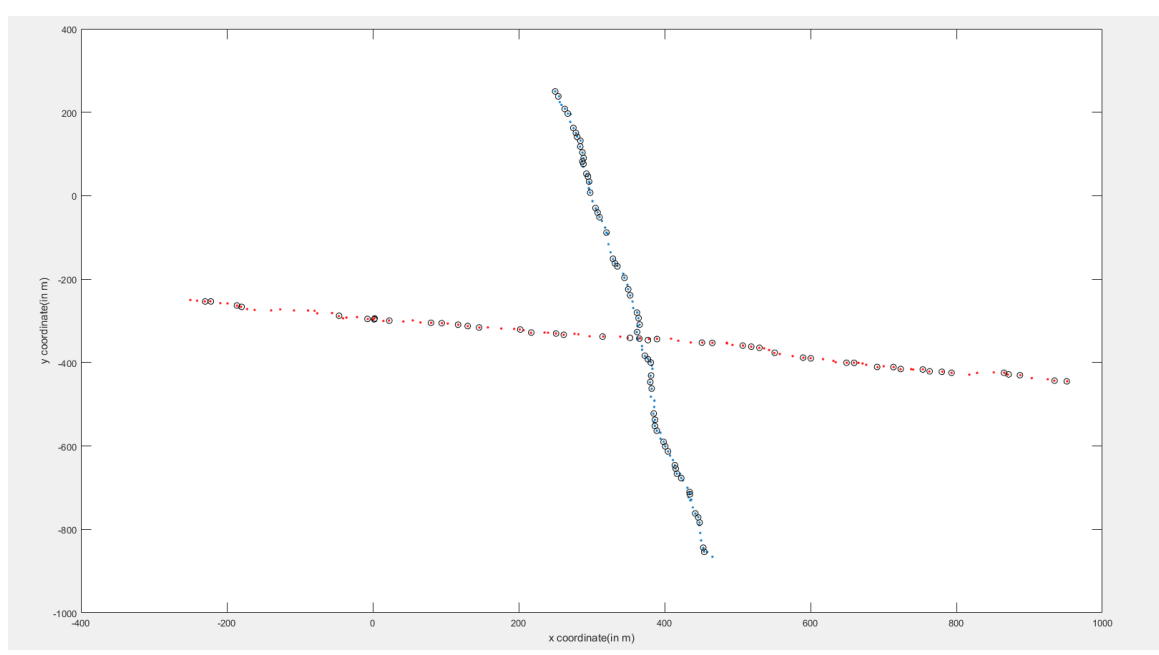

Figure 2. Algorithm Simulation of GM-PHD. 
$\mathrm{B}$ targets are moving at the speeds of $(2.5,-12)$ and $(12,-2)$, the deletion threshold is 4 . As shown in the figure, the traditional GM-PHD filter tracks the 2 targets A and B very well, and the calculation speed is much faster than the traditional PHD filter.

\section{Application of PHD Filter in Target Tracking}

In the past ten years, GM-PHD filtering has made a lot of research results under the continuous efforts of theoretical research scholars represented by Mahler and engineering technicians represented by Vo. At present, PHD filtering technology has been applied in many fields of research, extended multi-target tracking, image tracking, group target detection and tracking, multi-target tracking under unknown parameters and sensor management Numerous applications [5]. Next, we will summarize the outstanding achievements in various application fields.

- Multi-maneuvering target tracking: Vo applies GM-PHD filtering to multi-target tracking, proposes a multi-model PHD filtering method, and uses particle filtering to approximate the solution. Simulation shows that multi-target tracking is significantly better than single-model PHD filtering. Pasha et al. Also introduced the multi-model method into the GM-PHD filtering method, considering three kinds of motion models at the same time, and showed better performance than the traditional data association IMM-JPDA (Interacting Multiple Model) in tracking maneuvering targets.

- Expanded multi-target tracking: A model for measuring space recently published by Gilholm et al., Mahler extended PHD filtering to expanded multi-target tracking, obtained the expanded target PHD filter, and promoted the development of expanded multi-target tracking technology, but the algorithm Mahler Theoretical derivation was carried out, but no simulation was carried out. In 2010, on the basis of the GM-PHD filter proposed by Vo, Granstrom et al. They proposed that in the case of linear Gaussian, the Gaussian hybrid implementation form of expanding multiple target PHD filters was assumed, and the effectiveness of the filter was verified by simulation. Inspired by the multi-model method, Wang Xiao et al. They found an improved MM-GM-PHD to solve the problem of extended multi-target tracking, and the target has a certain maneuverability and achieved good results.

- Image tracking: Ikoma, Uchino, Maeda and others, they applied the particle PHD filter to the field of tracking feature points in optical images when time change. Clark, Vo, Bell and others applied the GM-PHD filter to the sonar image field, and effectively solved the problem of $2 \mathrm{D}$ or $3 \mathrm{D}$ active sonar image tracking. Wang et al. Applied the PHD filter with particles In the field of digital video images, it is used to solve the multi-target tracking problem.

- Group target detection and tracking issues: In the research practice of the situation assessment simulator system, the research team of the Swedish National Defense Research Agency applied the PHD filter to PHD group target tracking. A formation aggregation algorithm based on a multi-dimensional 
allocation method was used to detect the formation structure. And classification, an independent PHD filter is used for group target tracking detection on each formation structure. Clark et al. carried out research on group target tracking based on the GM-PHD filter method, by creating a target estimation state chart with an association relationship to represent the target group, used to limit the movement of each target Gaussian in its corresponding group, and through PHD Medium track maintenance method to identify target group tracks.

- Multi-target tracking under unknown parameters: Lian Feng et al. carried out multi-target tracking research under the background of unknown clutter. In 2009, an unknown hybrid clutter environment problem was proposed using Finite Mixture Models (FMM) and expectation poles. The method of estimating the clutter model by the Dahua algorithm. In 2010, a multi-target tracking method that satisfies the static conditions for the clutter distribution is proposed. Zhou Chengxing published a GM-PHD filtering method based on risk assessment in the case of unknown noise distribution.

- Sensor management: Mahler has proposed a multi-sensor multi-target management method based on PHD filtering. This method is based on the relative tactical priority of the target, which can achieve priority observation of key targets. EL-Fallah has verified this method in the research. In the simplified simulation scene, it can be realized by particle filtering method. Mahler and EL-Fallah put forward the formula for calculating feasible PENT in the study of the improved method of PHD filter, combined with the unknown dynamic clutter situation, and obtained a unified unknown dynamic clutter situation Sensor management method below. Because PHD filtering technology has broad prospects for solving the multi-target tracking problem, with the promotion of foreign research, more and more domestic research institutions and personnel are engaged in research and exploration in this field, mainly engaged in GM-PHD filtering implementation and GM -Research on the application of PHD filter.

\section{Prospect of PHD Filter Technology}

Throughout the above, PHD filtering has achieved many encouraging research results, focusing on the multi-target tracking research of PHD filtering, and now the existing problems in this field and the key aspects of future development are summarized as follows:

- Research on PHD filter

Implementation methods The current PHD filter implementation methods are mainly sequential Monte Carlo (particle filter) method and Gaussian mixture method, but the particle filter method requires higher selection of density function, and this type of function is difficult to confirm. A large number of sampling approximate calculations are required, which require high computing power and are difficult to implement in engineering. The Gaussian mixture me- 
thod requires linear Gaussian conditional assumptions. Although some researchers adopt approximate methods to deal with nonlinear non-Gaussian problems, the accuracy of target estimation has decreased. Therefore, it is necessary to carry out the algorithm research on the compromise between computational complexity and estimation accuracy.

- Target tracking in the case of multiple sensors

Research Currently, Target tracking in the case of multiple sensors based on PHD filtering usually assumes that the observation data of each sensor is completely independent, adopts sequential processing, and has low computational complexity, but low tracking accuracy and poor stability. The other is the product multi-sensor PHD filtering method, which comprehensively considers the multi-sensor observation information, which has better tracking accuracy and stability, but the calculation complexity is higher and it is difficult to achieve [6]. Therefore, how to achieve multi-sensor multi-target tracking with higher accuracy, stronger stability and easy engineering realization is the direction of future development.

- Research on the tracking method of multi-target situation

Currently, the tracking method of multi-target situation based on PHD filtering is mostly based on linear Gaussian models, which have certain limitations. It is more universal to carry out research on nonlinear non-Gaussian multi- extended target filtering methods [7]. The research on multi-expanded target tracking methods is mainly a two-dimensional model. If a three-dimensional extended model of an extended target can be developed, it will be able to describe the target more accurately and realistically, thereby obtaining more extended target information.

- Research on track generation

In the field of multi-target tracking, the PHD filter tracking algorithm can be used to obtain the number and status of the targets at any time. There is no track association information between adjacent target states, and the relationship between targets at consecutive moments is not given. Estimate the trajectory of all targets. At present, the track generation method based on PHD filtering is mainly combined with the traditional data correlation technology, and the multi-target RFS estimated at each discrete time is regarded as an "observation set", and then the traditional data correlation technology is used to form the target track, but this method does not Considering the situation of missed detection and false estimation of the target, it will lead to the formation of a false track and a target will produce multiple tracks, which will be detrimental to the comprehensive situation understanding and analysis of the surveillance scene [8]. In the future, how to realize joint state estimation and track generation and extraction under the framework of PHD filtering is a direction worthy of study.

- Research on multi-target joint detection and classification method

At present, most researches deal with the three problems of target detection, tracking and classification separately. However, these three problems can ac- 
tually be closely coupled. Joint processing is expected to achieve better detection, tracking and classification at the same time. Classification performance. At present, there are many researches on target JTC and target JDT, but there are not many studies on target joint JDTC methods, mainly because of the lack of an existing There is a theoretical framework for effective theoretical approaches to the foundation of strict theoretical interpretation, but the emergence of RFS theory fills the gap in this area, which can realize the simultaneous detection, tracking and classification of targets, which will greatly improve the ability of modern surveillance systems to acquire multi-target information, Has greater theoretical and practical significance.

\section{Acknowledgements}

This paper is funded by the International Exchange Program of Harbin Engineering University for Innovation-oriented Talents Cultivation. Thanks to my mentor for his longtime support and suggestions. Thanks for the support of my school.

\section{Conflicts of Interest}

The authors declare no conflicts of interest regarding the publication of this paper.

\section{References}

[1] Vo, B. and Ma, W. (2006) The Gaussian Mixture Probability Hypothesis Density Filter. IEEE Transactions on Signal Processing, 54, 4091-4104. https://doi.org/10.1109/TSP.2006.881190

[2] Vo, B., Clark, D.E., Vo, B. and Ristic, B. (2011) Bernoulli Forward-Backward Smoothing for Joint Target Detection and Tracking. IEEE Transactions on Signal Processing, 59, 4473-4477. https://doi.org/10.1109/TSP.2011.2158427

[3] Pasha, S.A., Vo, B., Tuan, H.D. and Ma, W. (2009) A Gaussian Mixture PHD Filter for Jump Markov System Models. IEEE Transactions on Aerospace and Electronic Systems, 45, 919-936. https://doi.org/10.1109/TAES.2009.5259174

[4] Mahler, R. (2009) PHD Filters for Nonstandard Targets, I: Extended Targets. 2009 12 th International Conference on Information Fusion, Seattle, WA, 915-921.

[5] Granstrom, K., Lundquist, C. and Orguner, O. (2012) Extended Target Tracking Using a Gaussian-Mixture PHD Filter. IEEE Transactions on Aerospace and Electronic Systems, 53, 1055-1058. https://doi.org/10.1109/TAES.2012.6324703

[6] Dehkordi, M.Y., Azimifar, Z. and Masnadishirazi, M.A. (2011) An Improvement on GM-PHD Filter for Occluded Target Tracking. 2011 IEEE International Conference on Acoustics, Speech and Signal Processing (ICASSP), Prague, 1773-1776. Wright and Wright, W. (1906) Flying-Machine. US Patent No. 821393. https://doi.org/10.1109/ICASSP.2011.5946846

[7] Edman, V., Andersson, M., Granström, K. and Gustafsson, F. (2013) Pedestrian Group Tracking Using the GM-PHD Filter. 21 st European Signal Processing Conference (EUSIPCO 2013), Marrakech, 1-5.

[8] Adeli, A., Yazdian-Dehkordi, M., Azimifar, Z. and Rojhani, O.R. (2012) Occluded Targets Tracking Using Improved GM-PHD Tracker. 2012 IEEE 11 th International Conference on Signal Processing, Beijing, 1071-1075. https://doi.org/10.1109/ICoSP.2012.6491763 